PROCEEDINGS OF THE

AMERICAN MATHEMATICAL SOCIETY

Volume 141, Number 9, September 2013, Pages 3213-3221

S 0002-9939(2013)11805-8

Article electronically published on June 3, 2013

\title{
AN EMBEDDING THEOREM
}

\section{N. A. CHERNYAVSKAYA AND L. A. SHUSTER}

(Communicated by Michael Hitrik)

Abstract. We consider a weighted space $W_{1}^{(2)}(\mathbb{R}, q)$ of Sobolev type:

$$
W_{1}^{(2)}(\mathbb{R}, q)=\left\{y \in A C_{\mathrm{loc}}^{(1)}(\mathbb{R}):\left\|y^{\prime \prime}\right\|_{L_{1}(\mathbb{R})}+\|q y\|_{L_{1}(\mathbb{R})}<\infty\right\},
$$

where $0 \leq q \in L_{1}^{\text {loc }}(\mathbb{R})$ and

$$
\|y\|_{W_{1}^{(2)}(\mathbb{R}, q)}=\left\|y^{\prime \prime}\right\|_{L_{1}(\mathbb{R})}+\|q y\|_{L_{1}(\mathbb{R})} .
$$

We obtain a precise condition which guarantees the embedding

$$
W_{1}^{(2)}(\mathbb{R}, q) \hookrightarrow L_{p}(\mathbb{R}), p \in[1, \infty) .
$$

\section{INTRODUCTION}

In the present paper, we consider a weighted function space $W_{1}^{(2)}(\mathbb{R}, q)$ of Sobolev type:

$$
W_{1}^{(2)}(\mathbb{R}, q)=\left\{y \in A C_{\mathrm{loc}}^{(1)}(\mathbb{R}):\left\|y^{\prime \prime}\right\|_{L_{1}(\mathbb{R})}+\|q y\|_{L_{1}(\mathbb{R})}<\infty\right\} .
$$

Here and in the sequel, $\|f\|_{p}=\|f\|_{L_{p}(\mathbb{R})}, p \in[1, \infty), A C_{\text {loc }}^{(1)}(\mathbb{R})$, is the set of functions absolutely continuous together with the derivative on every finite interval.

In this paper we always require that

$$
0 \leq q \in L_{1}^{\text {loc }}(\mathbb{R})
$$

and usually do not mention this requirement in the statements.

Our general goal is to find conditions under which there is an embedding

$$
W_{1}^{(2)}(\mathbb{R}, q) \hookrightarrow L_{p}(\mathbb{R}) .
$$

To be more precise, we give the following definitions.

Definition $1.1([6])$. Let $p \in[1, \infty)$. We say that the space $W_{1}^{(2)}(\mathbb{R}, q)$ is embedded into the space $L_{p}(\mathbb{R})$ (and write $W_{1}^{(2)}(\mathbb{R}, q) \hookrightarrow L_{p}(\mathbb{R})$ ) if $W_{1}^{(2)}(\mathbb{R}, q) \subseteq L_{p}(\mathbb{R})$ and

$$
\|y\|_{p} \leq c(p)\left\{\left\|y^{\prime \prime}\right\|_{1}+\|q y\|_{1}\right\}, \quad \forall y \in W_{1}^{(2)}(\mathbb{R}, q) .
$$

Our general convention is that the letters $c, c(\cdot)$ stand for absolute positive constants which are not essential for exposition and may differ even within a single chain of calculations.

We now state our main result.

Received by the editors December 1, 2011.

2010 Mathematics Subject Classification. Primary 46E35; Secondary 34B24.

Key words and phrases. Embedding theorem. 
Theorem 1.2. Let $p \in[1, \infty)$. Then the embedding $W_{1}^{(2)}(\mathbb{R}, q) \hookrightarrow L_{p}(\mathbb{R})$ takes place if and only if

$$
\exists a>0: \quad q_{0}(a) \stackrel{\text { def }}{=} \inf _{x \in \mathbb{R}} \int_{x-a}^{x+a} q(t) d t>0 .
$$

Note that for $p=1$ this assertion was proved in [4] and will not be reconsidered here (see $\S 2)$.

\section{Example 1.3. Let}

$$
q(x)=1+\cos \left(|x|^{\theta}\right), \quad x \in \mathbb{R}, \theta \in(0, \infty) .
$$

In 3 it was shown that in case (1.5) condition (1.4) holds if and only if $\theta \geq 1$. Together with Theorem 1.2 this implies that in case (1.5) the embedding $W_{1}^{(2)}(\mathbb{R}, q) \hookrightarrow$ $L_{p}(\mathbb{R}), p \in[1, \infty)$, takes place if and only if $\theta \geq 1$.

\section{Preliminaries}

In this section, we present some facts needed for the proofs (see $\S 3$ ).

Lemma 2.1 (11, 2]). Suppose conditions (1.2) and (2.1) hold:

$$
\int_{-\infty}^{x} q(t) d t>0, \quad \int_{x}^{\infty} q(t) d t>0, \quad \forall x \in \mathbb{R} .
$$

Then there exists a fundamental system of solutions (FSS) $\{u, v\}$ of the equation

$$
z^{\prime \prime}(x)=q(x) z(x), \quad x \in \mathbb{R},
$$

which has the following properties:

$$
\begin{gathered}
u(x)>0, \quad v(x)>0, \quad u^{\prime}(x) \leq 0, \quad v^{\prime}(x) \geq 0, \quad \forall x \in \mathbb{R}, \\
v^{\prime}(x) u(x)-u^{\prime}(x) v(x)=1, \quad \forall x \in \mathbb{R}, \\
\lim _{x \rightarrow-\infty} \frac{v(x)}{u(x)}=\lim _{x \rightarrow \infty} \frac{u(x)}{v(x)}=0 .
\end{gathered}
$$

The solutions $\{u, v\}$ and the function $\rho$,

$$
\rho(x)=u(x) v(x), \quad x \in \mathbb{R},
$$

satisfy the relations

$$
\begin{gathered}
\int_{-\infty}^{0} \frac{d t}{\rho(t)}=\int_{0}^{\infty} \frac{d t}{\rho(t)}=\infty, \\
\left|\rho^{\prime}(x)\right|<1, \quad \forall x \in \mathbb{R}, \\
\frac{v^{\prime}(x)}{v(x)}=\frac{1+\rho^{\prime}(x)}{2 \rho(x)}, \quad \frac{u^{\prime}(x)}{u(x)}=-\frac{1-\rho^{\prime}(x)}{2 \rho(x)}, \quad x \in \mathbb{R} .
\end{gathered}
$$

In addition, if $G(x, t)$ is the Green function,

$$
G(x, t)= \begin{cases}u(x) v(t), & x \geq t \\ u(t) v(x), & x \leq t\end{cases}
$$

corresponding to the equation

$$
-y^{\prime \prime}(x)+q(x) y(x)=f(x), \quad x \in \mathbb{R},
$$


then we have the inequality

$$
\sup _{t \in \mathbb{R}} \Phi(t) \leq 1, \quad \Phi(t)=\int_{-\infty}^{\infty} q(\xi) G(t, \xi) d \xi, \quad t \in \mathbb{R} .
$$

Finally, for every $p \in[1, \infty)$, equation (2.2) has no solutions $z \in L_{p}(\mathbb{R})$ apart from $z \equiv 0$.

Lemma 2.2 ([1]). Suppose conditions (1.2) and (2.1) hold. For a given $x \in \mathbb{R}$, consider the equation in $d \geq 0$ :

$$
d \int_{x-d}^{x+d} q(t) d t=2
$$

Equation (2.13) has a unique finite positive solution for every $x \in \mathbb{R}$. Denote this solution by $d(x)$. The function $d(x)$ is continuous for $x \in \mathbb{R}$.

Note that the function $d$ was introduced by M. Otelbaev (see [6]).

Theorem 2.3 (1]). Suppose conditions (1.2) and (2.1) hold. Then we have the inequalities

$$
\frac{d(x)}{4} \leq \rho(x) \leq \frac{3}{2} d(x), \quad x \in \mathbb{R} .
$$

Estimates of type (2.14) were first obtained (under some additional requirements to the function $q$ ) by Otelbaev (see [7]) and are therefore called Otelbaev's inequalities (see also [6]).

Consider equation (2.11) with $f \in L_{p}(\mathbb{R}), p \in[1, \infty)$. Below by a solution of (2.11) we mean any function $y \in A C_{\text {loc }}^{(1)}(\mathbb{R})$ satisfying equality (2.11) almost everywhere on $\mathbb{R}$.

Definition $2.4([3])$. We say that equation (2.11) is correctly solvable in the space $L_{p}(\mathbb{R}), p \in[1, \infty)$, if assertions I-II hold:

I) for any function $f \in L_{p}(\mathbb{R})$, there is a unique solution $y \in L_{p}(\mathbb{R})$ of (2.11);

II) the solution $y \in L_{p}(\mathbb{R})$ of (2.11) satisfies the estimate

$$
\|y\|_{p} \leq c(p)\|f\|_{p}, \quad \forall f \in L_{p}(\mathbb{R}) .
$$

Theorem $2.5([3])$. Let $p \in[1, \infty)$. Equation (2.11) is correctly solvable in $L_{p}(\mathbb{R})$ if and only if one of the following two equivalent conditions, A, B, holds:

A) One has inequalities (2.1) and $d_{0}<\infty$. Here

$$
d_{0}=\sup _{x \in \mathbb{R}} d(x) .
$$

B) There is $a \in(0, \infty)$ such that $q_{0}(a)>0$ :

$$
q_{0}(a)=\inf _{x \in \mathbb{R}} \int_{x-a}^{x+a} q(t) d t .
$$

In addition, if equation (2.11) is correctly solvable in $L_{p}(\mathbb{R}), p \in[1, \infty)$, then its solution $y \in L_{p}(\mathbb{R})$ is of the form

$$
y(x)=(G f)(x) \stackrel{\text { def }}{=} \int_{-\infty}^{\infty} G(x, t) f(t) d t, \quad x \in \mathbb{R} .
$$

Theorem 2.6 ([4). The embedding $W_{1}^{(2)}(\mathbb{R}, q) \hookrightarrow L_{1}(\mathbb{R})$ takes place if and only if equation (2.11) is correctly solvable in $L_{1}(\mathbb{R})$. 
Theorem 2.7 ([5, Ch. XI, §6]). Let $K(x, t)$ be a continuous positive function in variables $x, t \in \mathbb{R}$, and let $K$ be the integral operator

$$
(K f)(x)=\int_{-\infty}^{\infty} K(x, t) f(t) d t, \quad \forall f \in L_{1}(\mathbb{R}) .
$$

Then for every $p \in(1, \infty)$, we have the equality

$$
\|K\|_{L_{1}(\mathbb{R}) \rightarrow L_{p}(\mathbb{R})}=\sup _{t \in \mathbb{R}}\left(\int_{-\infty}^{\infty} K(x, t)^{p} d x\right)^{1 / p} .
$$

\section{ProOFs}

Proof of Theorem 1.2. Necessity.

Lemma 3.1. Let $W_{1}^{(2)}(\mathbb{R}, q) \hookrightarrow L_{p}(\mathbb{R}), p \in[1, \infty)$. Then relations (2.1) hold.

Proof. Assume the contrary, say,

$$
\int_{x_{0}}^{\infty} q(t) d t=0
$$

for some $x_{0} \in \mathbb{R}$. Let $\varphi \in C^{\infty}(\mathbb{R})$ be such that $\operatorname{supp} \varphi=\left[x_{0}, \infty\right), 0 \leq \varphi \leq 1$, for $x \in \mathbb{R}$ and $\varphi(x) \equiv 1$ for $x \geq x_{0}+1$. Clearly, $\varphi \in A C_{\text {loc }}^{(1)}(\mathbb{R})$ and, in addition,

$$
\begin{gathered}
\int_{-\infty}^{\infty}|q(t) \varphi(t)| d t=\int_{x_{0}}^{\infty} q(t) \varphi(t) d t=0, \\
0<\int_{-\infty}^{\infty}\left|\varphi^{\prime \prime}(t)\right| d t=\int_{x_{0}}^{x_{0}+1}\left|\varphi^{\prime \prime}(t)\right| d t=c<\infty .
\end{gathered}
$$

Hence $\varphi \in W_{1}^{(2)}(\mathbb{R}, q)$, and since $W_{1}^{(2)}(\mathbb{R}, q) \hookrightarrow L_{p}(\mathbb{R})$, we have

$$
\begin{aligned}
\infty & >c\left\{\int_{-\infty}^{\infty}\left|\varphi^{\prime \prime}(t)\right| d t+\int_{-\infty}^{\infty}|q(t) \varphi(t)| d t\right\} \geq\left(\int_{-\infty}^{\infty}|\varphi(t)|^{p} d t\right) \geq\left(\int_{x_{0}+1}^{\infty} 1 d t\right)^{1 / p} \\
& =\infty
\end{aligned}
$$

Contradiction.

Below, conditions (1.2) and (2.1) are assumed to be valid and do not appear in the statements.

Lemma 3.2. For a given $x \in \mathbb{R}$, consider the equation in $s \geq 0$ :

$$
\int_{x-s}^{x+s} \frac{d t}{d(t)}=2
$$

Equation (3.1) has a unique finite positive solution for every $x \in \mathbb{R}$. Denote it by $s(x)$. Then for every $t \in[x-s(x), x+s(x)]$ and $x \in \mathbb{R}$, we have the inequalities

$$
\begin{aligned}
& c^{-1} \leq \frac{u(t)}{u(x)}, \quad \frac{v(t)}{v(x)} \leq c, \\
& c^{-1} d(x) \leq s(x) \leq c d(x) .
\end{aligned}
$$


Proof. Consider the function

$$
F(s)=\int_{x-s}^{x+s} \frac{d \xi}{d(\xi)}, \quad s \geq 0
$$

Clearly, $F(0)=0$, the function $F(s)$ is monotone increasing and continuous on $[0, \infty)$, and, in addition, in view of (2.7) and (2.14), we have

$$
F(s)=\int_{x-s}^{x+s} \frac{d t}{d(t)} \geq \frac{1}{4} \int_{x-s}^{x+s} \frac{d t}{\rho(t)} \rightarrow \infty \quad \text { as } \quad s \rightarrow \infty .
$$

Hence, for a given $x \in \mathbb{R}$, equation (3.1) has a unique finite positive solution $s(x)$. Further, from (2.8), (2.9) and (2.14), it follows that

$$
\frac{v^{\prime}(\xi)}{v(\xi)}=\frac{1+\rho^{\prime}(\xi)}{2 \rho(\xi)} \leq \frac{1}{\rho(\xi)} \leq \frac{4}{d(\xi)}, \quad \xi \in \mathbb{R} .
$$

For $t \in[x, x+s(x)]$ this implies that

$$
\ln \frac{v(t)}{v(x)} \leq 4 \int_{x}^{t} \frac{d \xi}{d(\xi)} \leq 4 \int_{x-s(x)}^{x+s(x)} \frac{d \xi}{d(\xi)}=8 .
$$

According to (2.3), this gives

$$
c^{-1} \leq 1 \leq \frac{v(t)}{v(x)} \leq c \quad \text { for } \quad t \in[x, x+s(x)], \quad x \in \mathbb{R} .
$$

Similarly, if $t \in[x-s(x), x]$, then

$$
\begin{gathered}
\ln \frac{v(x)}{v(t)} \leq 4 \int_{t}^{x} \frac{d \xi}{d(\xi)} \leq 4 \int_{x-s(x)}^{x+s(x)} \frac{d \xi}{d(\xi)}=8 \\
\Rightarrow c^{-1} \leq 1 \leq \frac{v(x)}{v(t)} \leq c \quad \text { for } \quad t \in[x-s(x), x], \quad x \in \mathbb{R} .
\end{gathered}
$$

From (3.5) and (3.6) we obtain (3.2) for $v$ (inequalities (3.2) for $u$ are proved in a similar way). Further, by (3.1), (3.2) and (2.14), we get

$$
\begin{aligned}
2 & =\int_{x-s(x)}^{x+s(x)} \frac{d \xi}{d(\xi)}=\int_{x-s(x)}^{x+s(x)} \frac{\rho(\xi)}{d(\xi)} \cdot \frac{u(x)}{u(\xi)} \cdot \frac{v(x)}{v(\xi)} \cdot \frac{d(x)}{\rho(x)} \cdot \frac{d \xi}{d(x)} \geq \frac{1}{c} \cdot \frac{s(x)}{d(x)} \\
2 & =\int_{x-s(x)}^{x+s(x)} \frac{d \xi}{d(\xi)}=\int_{x-s(x)}^{x+s(x)} \frac{\rho(\xi)}{d(\xi)} \cdot \frac{u(x)}{u(\xi)} \cdot \frac{v(x)}{v(\xi)} \cdot \frac{d(x)}{\rho(x)} \cdot \frac{d \xi}{d(x)} \leq c \frac{s(x)}{d(x)}
\end{aligned}
$$

Lemma 3.3. Suppose conditions (1.2) and (2.1) hold. Set

$$
\begin{gathered}
f_{x}(t)= \begin{cases}1, & \text { if } t \in[x-s(x), x+s(x)], \\
0, & \text { if } t \notin[x-s(x), x+s(x)],\end{cases} \\
\left.y_{x}(t)=\int_{-\infty}^{\infty} G(t, \xi) f_{x}(\xi) d \xi, \quad t \in \mathbb{R} \quad \text { (see (2.10) }\right) .
\end{gathered}
$$

Then we have the relations

$$
\begin{gathered}
-\left(y_{x}(t)\right)^{\prime \prime}+q(t) y_{x}(t)=f(t), \quad t \in \mathbb{R}, \\
\left\|\left(y_{x}\right)^{\prime \prime}\right\|_{1}+\left\|q y_{x}\right\|_{1} \leq \operatorname{cs}(x), \quad x \in \mathbb{R} .
\end{gathered}
$$


Proof. From (2.10) and (3.8) it follows that

$$
y_{x}(t)=u(t) \int_{-\infty}^{t} v(\xi) f_{x}(\xi) d \xi+v(t) \int_{t}^{\infty} u(\xi) f_{x}(\xi) d \xi .
$$

According to (3.7), integration in (3.11) is along segments which are contained in the finite segment $[x-s(x), x+s(x)]$, and therefore the function $y_{x}$ is well-defined. Equality (3.9) can be checked directly using Lemma 2.1. Further, from Fubini's Theorem and (2.12), it follows that

$$
\begin{aligned}
\|q y\|_{1} & =\int_{-\infty}^{\infty} q(t)\left|\int_{-\infty}^{\infty} G(t, \xi) f_{x}(\xi) d \xi\right| d t=\int_{-\infty}^{\infty} q(t) \int_{-\infty}^{\infty} G(t, \xi) f_{x}(\xi) d \xi d t \\
2) & =\int_{-\infty}^{\infty} f_{x}(\xi)\left[\int_{-\infty}^{\infty} q(t) G(t, \xi) d t\right] d \xi \leq \int_{-\infty}^{\infty} f_{x}(\xi) d \xi=2 s(x) .
\end{aligned}
$$

Estimate (3.10) follows from (3.12), (3.7), (3.9) and the triangle inequality for norms.

Corollary 3.4. Suppose conditions (1.2) and (2.1) hold. Let $x \in \mathbb{R}$, and let $y_{x}(t)$, $t \in \mathbb{R}$, be the function defined in (3.8). Then we have the inequalities

$$
\begin{gathered}
y_{x}(t) \geq c^{-1} s^{2}(x) \quad \text { if } \quad|t-x| \leq s(x), \\
\left\|y_{x}\right\|_{p} \geq c^{-1} s(x)^{2+1 / p}, \quad x \in \mathbb{R}, \quad p \in[1, \infty) .
\end{gathered}
$$

Proof. Now we use (3.11), (3.2), (3.3) and (2.14):

$$
\begin{aligned}
y_{x}(t) & =u(t) \int_{x-s(x)}^{t} v(\xi) d \xi+v(t) \int_{t}^{x+s(x)} u(\xi) d \xi \\
& =\rho(x)\left\{\frac{u(t)}{u(x)} \int_{x-s(x)}^{t} \frac{v(\xi)}{v(x)} d \xi+\frac{v(t)}{v(x)} \int_{t}^{x+s(x)} \frac{u(\xi)}{u(x)} d \xi\right\} \\
& \geq \rho(x) s(x) c^{-1} \geq c^{-1} d(x) s(x) \geq c^{-1} s^{2}(x) \Rightarrow(3.13) .
\end{aligned}
$$

Estimate (3.14) follows from (3.13):

$$
\left\|y_{x}\right\|_{p} \geq\left[\int_{x-s(x)}^{x+s(x)}\left|y_{x}(t)\right|^{p} d t\right]^{1 / p} \geq c^{-1} s(x)^{2+1 / p} .
$$

Let us now go to the proof of the theorem. Let $p \in(1, \infty)$, and let $W_{1}^{(2)}(\mathbb{R}, q) \hookrightarrow$ $L_{p}(\mathbb{R})$. According to the facts proven above, we have (2.1), the functions $d, s$ and $y_{x}($ see (3.8) $)$ are defined, and $y_{x} \in W_{1}^{(2)}(\mathbb{R}, q)$ in view of (3.10). This implies that

$$
c s(x) \geq\left\|\left(y_{x}\right)^{\prime \prime}\right\|_{1}+\left\|q y_{x}\right\|_{1} \geq c^{-1}\left\|y_{x}\right\|_{p} \geq c^{-1} s(x)^{2+1 / p} .
$$

Hence $s(x) \leq c<\infty, x \in \mathbb{R}$, and therefore $d_{0}<\infty$ (see (3.3), (2.16)). Then $q_{0}(a)>0$ for some $a \in(0, \infty)$ (see Theorem 2.5).

Proof of Theorem 1.2, Sufficiency.

Lemma 3.5. Suppose conditions (1.2) and (2.1) hold. Then for every $x \in \mathbb{R}$, we have the equality

$$
4 \sqrt{\rho(x)}=\int_{-\infty}^{\infty} \frac{G(x, \xi) d \xi}{\rho(\xi)^{3 / 2}}
$$


Proof. The following elementary relations follow from Lemma 2.1

$$
\begin{aligned}
\left(\sqrt{\frac{v(\xi)}{u(\xi)}}\right)^{\prime} & =\frac{1}{2} \sqrt{\frac{u(\xi)}{v(\xi)}} \frac{v^{\prime}(\xi) u(\xi)-u^{\prime}(\xi) v(\xi)}{u^{2}(\xi)} \\
& =\frac{1}{2 \sqrt{v(\xi)}} \frac{1}{u(\xi)^{3 / 2}}=\frac{1}{2} \frac{v(\xi)}{\rho(\xi)^{3 / 2}}, \quad \xi \in \mathbb{R} \\
& \Rightarrow \sqrt{\frac{v(x)}{u(x)}}-\sqrt{\frac{v(t)}{u(t)}}=\frac{1}{2} \int_{t}^{x} \frac{v(\xi) d \xi}{\rho(\xi)^{3 / 2}}, \quad t \leq x \\
& \Rightarrow \sqrt{\frac{v(x)}{u(x)}}=\sqrt{\frac{v(x)}{u(x)}}-\lim _{t \rightarrow-\infty} \sqrt{\frac{v(t)}{u(t)}}=\frac{1}{2} \int_{-\infty}^{x} \frac{v(\xi) d \xi}{\rho(\xi)^{3 / 2}} \\
& \Rightarrow \sqrt{\rho(x)}=\frac{1}{2} u(x) \int_{-\infty}^{x} \frac{v(\xi) d \xi}{\rho(\xi)^{3 / 2}}=\frac{1}{2} \int_{-\infty}^{x} \frac{G(x, \xi) d \xi}{\rho(\xi)^{3 / 2}}, \quad x \in \mathbb{R} .
\end{aligned}
$$

Similarly, one can check the equality

$$
\sqrt{\rho(x)}=\frac{1}{2} \int_{x}^{\infty} \frac{G(x, \xi) d \xi}{\rho(\xi)^{3 / 2}}, \quad x \in \mathbb{R} .
$$

The latter two relations imply (3.15).

Let us introduce the notation

$$
H_{p}=\sup _{x \in \mathbb{R}} \int_{-\infty}^{\infty} G(x, t)^{p} d t, \quad p \in[1, \infty) .
$$

Lemma 3.6. Suppose conditions (1.2) and (2.1) hold. Then we have the inequalities (see (2.16) )

$$
c^{-1} d_{0}^{2} \leq H_{1} \leq c d_{0}^{2} .
$$

Proof. Let $x \in \mathbb{R}$. Now we use Lemmas 2.2, 3.2, and the estimate (3.13):

$$
\begin{aligned}
\int_{-\infty}^{\infty} G(x, t) d t & \geq \int_{x-s(x)}^{x+s(x)} G(x, t) d t=u(x) \int_{x-s(x)}^{x} v(t) d t+v(x) \int_{x}^{x+s(x)} u(t) d t \\
& \geq c^{-1} s^{2}(x) \geq c^{-1} d^{2}(x) \quad \Rightarrow \quad H_{1} \geq c^{-1} d_{0}^{2} .
\end{aligned}
$$

In particular, if $d_{0}=\infty$, then from the facts proven above it follows that $H_{1}=\infty$, and therefore in this case the upper estimate in (3.17) also holds. Now let $d_{0}<\infty$. In the following relations, we use (3.15) and (2.14):

$$
\begin{aligned}
4 \sqrt{d_{0}} & \geq 4 \sqrt{d(x)} \geq c^{-1} \sqrt{\rho(x)}=\frac{c^{-1}}{4} \int_{-\infty}^{\infty} \frac{G(x, \xi) d \xi}{\rho(\xi)^{3 / 2}} \\
& \geq c^{-1} \int_{-\infty}^{\infty} \frac{G(x, \xi) d \xi}{d(\xi)^{3 / 2}} \geq \frac{c^{-1}}{d_{0}^{3 / 2}} \int_{-\infty}^{\infty} G(x, \xi) d \xi, \quad x \in \mathbb{R} .
\end{aligned}
$$

Hence $H_{1} \leq c d_{0}^{2}$, as required.

Corollary 3.7. Suppose conditions (1.2) and (2.1) hold, and let $p \in(1, \infty)$. Then we have the inequalities

$$
c^{-1}(p) d_{0}^{p+1} \leq H_{p} \leq c(p) d_{0}^{p+1} .
$$


Proof. The lower estimate in (3.18) is proved exactly as the lower estimate in (3.17). Here, if $d_{0}=\infty$, then also $H_{p}=\infty$, and then the upper estimate in (3.18) also holds. Now let $d_{0}<\infty$. The following relations are based on Lemma 2.1, (2.14) and (3.17):

$$
\begin{aligned}
\int_{-\infty}^{\infty} G(x, t)^{p} d t & =u^{p}(x) \int_{-\infty}^{x} v^{p}(t) d t+v^{p}(x) \int_{x}^{\infty} u(t)^{p} d t \\
& \leq u^{p}(x) v^{p-1}(x) \int_{-\infty}^{x} v(t) d t+v(x)^{p} u(x)^{p-1} \int_{x}^{\infty} u(t) d t \\
& =\rho(x)^{p-1} \int_{-\infty}^{\infty} G(x, t) d t \leq c_{p} d(x)^{p-1} d_{0}^{2} \leq c(p) d_{0}^{p+1}
\end{aligned}
$$

This gives $H_{p} \leq c(p) d_{0}^{p+1}$.

Let us now go to the proof of the theorem. Let $q_{0}(a)>0$ for some $a \in(0, \infty)$, and let $y \in W_{1}^{(2)}(\mathbb{R}, q)$. Denote

$$
f(x)=-y^{\prime \prime}(x)+q(x) y(x), \quad x \in \mathbb{R} .
$$

Then, clearly, $f \in L_{1}(\mathbb{R})$ since

$$
\|f\|_{1} \leq\left\|y^{\prime \prime}\right\|_{1}+\|q y\|_{1}<\infty .
$$

Besides, since $W_{1}^{(2)}(\mathbb{R}, q) \hookrightarrow L_{1}(\mathbb{R})$ (see Theorems 2.5 and 2.6), in view of the embedding, we have

$$
\|y\|_{1} \leq c\left\{\left\|y^{\prime \prime}\right\|_{1}+\|q y\|_{1}\right\}<\infty .
$$

Let us now consider the equation

$$
-\tilde{y}^{\prime \prime}+q(x) \tilde{y}(x)=f(x), \quad x \in \mathbb{R},
$$

where the function $f$ is defined according to (3.19). By Theorem 2.5, equation (2.11) is correctly solvable in the space $L_{1}(\mathbb{R})$, and therefore (3.22) has a unique solution $\tilde{y} \in L_{1}(\mathbb{R})$, and

$$
\|\tilde{y}\|_{1} \leq c\|f\|_{1} \leq c\left\{\left\|y^{\prime \prime}\right\|_{1}+\|q y\|_{1}\right\}<\infty .
$$

From (3.22) and (3.19) it follows that the function

$$
z(x)=\tilde{y}(x)-y(x), \quad x \in \mathbb{R},
$$

is a solution of (2.2), and $z \in L_{1}(\mathbb{R})$ in view of (3.21) and (3.23). Hence $z \equiv 0$ by Lemma 2.1 and $y \equiv \tilde{y}$. Then from (2.18) we get

$$
y(x) \equiv \tilde{y}(x)=\int_{-\infty}^{\infty} G(x, t) f(t) d t, \quad x \in \mathbb{R},
$$

where the function $f$ is defined by (3.19). Since $f \in L_{1}(\mathbb{R})$ (see (3.20)) and, by Theorem 2.7. Corollary 3.7 and Theorem 2.5 , the operator $G: L_{1}(\mathbb{R}) \rightarrow L_{p}(\mathbb{R})$ is bounded, we obtain from (3.24) that

$$
\|y\|_{p}=\|G f\|_{p} \leq\|G\|_{L_{1}(\mathbb{R}) \rightarrow L_{p}(\mathbb{R}} \cdot\|f\|_{1} \leq c(p) d_{0}^{1+1 / p}\left\{\left\|y^{\prime \prime}\right\|_{1}+\|q y\|_{1}\right\},
$$

as required. 


\section{REFERENCES}

[1] N. Chernyavskaya and L. Shuster, Estimates for the Green function of a general SturmLiouville operator and their applications, Proc. Amer. Math. Soc. 127 (1999), 1413-1426. MR1625725 (99h:34040)

[2] N. Chernyavskaya and L. Shuster, Regularity of the inversion problem for the Sturm-Liouville equation in the spaces $L_{p}$, Methods and Applications of Analysis 7 (1) (2000), 65-84. MR.1796006 (2001i:34010)

[3] N. Chernyavskaya and L. Shuster, A criterion for correct solvability of the Sturm-Liouville equation in the space $L_{p}(R)$, Proc. Amer. Math. Soc 130 (2001), 1043-1054. MR.1873778 (2002j:34040)

[4] N. A. Chernyavskaya and L. Shuster, An embedding theorem for a weighted space of Sobolev type and correct solvability of the Sturm-Liouville equation, Czechoslovak Math. J. 62 (2012), no. 3, 709-716. MR2984630

[5] L.W. Kantorovich and G.P. Akilov, Functional Analysis, Nauka, Moscow, 1977. MR.0511615 $(58: 23465)$

[6] K. Mynbaev and M. Otelbaev, Weighted Function Spaces and the Spectrum of Differential Operators, Nauka, Moscow, 1988. MR950172 (89h:46036)

[7] M. Otelbaev, A criterion for the resolvent of a Sturm-Liouville operator to be a kernel, Math. Notes 25 (1979), 296-297 (translation of Mat. Zametki). MR534299 (80k:34037)

Department of Mathematics and Computer Science, Ben-Gurion University of the Negev, P.O. Box 653, Beer-Sheva, 84105, Israel

Department of Mathematics, Bar-Ilan University, 52900 Ramat Gan, Israel

E-mail address: miriam@macs.biu.ac.il 